\title{
Dynamic magnetic resonance imaging in assessing lung function in adolescent idiopathic scoliosis: a pilot study of comparison before and after posterior spinal fusion Winnie CW Chu*1, Bobby KW Ng², Albert $\mathrm{M} \mathrm{Li}^{3}$, Tsz-ping Lam², Wynnie WM Lam ${ }^{1}$ and Jack CY Cheng ${ }^{2}$
}

Address: ${ }^{1}$ Departments of Diagnostic Radiology and Organ Imaging, The Chinese University of Hong Kong, Prince of Wales Hospital, Shatin, Hong Kong, China, ${ }^{2}$ Departments of Orthopaedics and Traumatology, The Chinese University of Hong Kong, Prince of Wales Hospital, Shatin, Hong Kong, China and ${ }^{3}$ Departments of Paediatrics, The Chinese University of Hong Kong, Prince of Wales Hospital, Shatin, Hong Kong, China

Email: Winnie CW Chu* - winnie@med.cuhk.edu.hk; Bobby KW Ng - bobng@ort.cuhk.edu.hk; Albert M Li - albertmli@cuhk.edu.hk; Tszping Lam - tplam@ort.cuhk.edu.hk; WynnieWM Lam - wynnie@cuhk.edu.hk; Jack CY Cheng - jackcheng@cuhk.edu.hk

* Corresponding author

Published: 19 November 2007

Journal of Orthopaedic Surgery and Research 2007, 2:20 doi:10.1186/1749-799X-2-20
Received: 27 January 2007

Accepted: 19 November 2007

This article is available from: http://www.josr-online.com/content/2/I/20

() 2007 Chu et al; licensee BioMed Central Ltd.

This is an Open Access article distributed under the terms of the Creative Commons Attribution License (http://creativecommons.org/licenses/by/2.0), which permits unrestricted use, distribution, and reproduction in any medium, provided the original work is properly cited.

\begin{abstract}
Background: Restrictive impairment is the commonest reported pulmonary deficit in AIS, which improves following surgical operation. However, exact mechanism of how improvement is brought about is unknown. Dynamic fast breath-hold (BH)-MR imaging is a recent advance which provides direct quantitative visual assessment of pulmonary function. By using above technique, change in lung volume, chest wall and diaphragmatic motion in AIS patients before and six months after posterior spinal fusion surgery were measured.
\end{abstract}

Methods: 16 patients with severe right-sided predominant thoracic scoliosis (standing Cobb's angle $50^{\circ}-82^{\circ}$, mean $60^{\circ}$ ) received posterior spinal fusion without thoracoplasty were recruited into this study. BH-MR sequences were used to obtain coronal images of the whole chest during full inspiration and expiration. The following measurements were assessed: (I) inspiratory, expiratory and change in lung volume; (2) change in anteroposterior (AP) and transverse (TS) diameter of the chest wall at two levels: carina and apex (3) change in diaphragmatic heights. The changes in parameters before and after operation were compared using Wilcoxon signed ranks test. Patients were also asked to score their breathing effort before and after operation using a scale of $\mathrm{I}-9$ with ascending order of effort. The degree of spinal surgical correction at three planes was also assessed by reformatted MR images and correction rate of Cobb's angle was calculated.

Results: The individual or total inspiratory and expiratory volume showed slight but insignificant increase after operation. There was significantly increase in bilateral TS chest wall movement at carina level and increase in bilateral diaphragmatic movements between inspiration and expiration. The AP chest wall movements, however, did not significantly change.

The median breathing effort after operation was lower than that before operation $(p<0.05)$.

There was significant reduction in coronal Cobb's angle after operation but the change in sagittal and axial angle at scoliosis apex was not significant.

Conclusion: There is improvement of lateral chest wall and diaphragmatic motions in AIS patients six months after posterior spinal fusion, associated with subjective symptomatic improvement. Lung volumes however, do not significantly change after operation. BH-MR is novel non-invasive method for long term post operative assessment of pulmonary function in AIS patients. 


\section{Background}

Adolescent idiopathic scoliosis (AIS) is the most common form of idiopathic scoliosis, typically affecting growing adolescent girls, 10-16 years of age. Untreated scoliosis has an increased risk of developing respiratory failure and premature mortality[1]. Pulmonary function impairment in AIS patients might be related to restricted lung volume, poor chest wall expansibility or impaired diaphragmatic motion. Little is known about which of the above factors is more significantly correlated with the pulmonary deficit in AIS. Restrictive impairment is the commonest reported pulmonary deficit in AIS, which improves following surgical operation[2,3]. However, the exact mechanism of how the improvement is brought about is unknown.

We have previously reported a validated novel imaging technique for assessment of pulmonary function in AIS subjects. Kotani and colleagues have also investigated on chest wall and diaphragmatic movement of scoliosis patients using dynamic breathing MRI [4]. With the application of ultrafast dynamic breath-hold (BH) MR imaging and multiplanar reformat technique, the lung volume, chest wall, and diaphragmatic motions between inspiration and expiration can be accurately measured with high reproducibility in both AIS subjects and normal controls[5].

The aim of this pilot study was to evaluate the change in lung volume, chest wall and diaphragmatic motion in AIS patients before operation and six months after posterior spinal fusion surgery.

\section{Methods \\ Subjects}

The study included 16 idiopathic scoliosis girls with a predominant right-sided thoracic curve (Standing Cobb's angle ranged from $50^{\circ}-82^{\circ}$, mean $60^{\circ}$ ). The distribution of curves by Lenke classification is given in Table 1 . Their age ranged from 11-18 (mean 14.4). They were consecutively included for scheduled posterior spinal fusion without thoracoplasty from 2002 to 2004. The surgical procedure was performed under SSEP monitoring. It consisted of a standard midline posterior exposure, subperio-

Table I: Frequency of curve types by Lenke classification in 16 subjects included in this study

\begin{tabular}{ll}
\hline Lenke's classification & Frequency \\
\hline IA- & 1 \\
IAN & 6 \\
IBN & 5 \\
IC- & 1 \\
$2 A-$ & 1 \\
$3 A N$ & 1 \\
BBN & 1 \\
\hline
\end{tabular}

steal dissection made from spinous process to the tips of each transverse process. The facet capsules were meticulously elevated till the superior rib surface was reached. Partial excision of the ligamentum flavum was made between spinous processes for the lordotic thoracic segments typically from T5 to T11. The exposure was made to preserve vascularity to paraspinal muscles. Instrumentation consisted of three types. (a) 3 cases of Harrington rod on concave side, Luque rod on convex side and Wisconsin wire for the instrumented spinous processes. (b) 8 cases of ISOLA instrumentation consisted of pedicle screws to lumbar segments to build a base, proximal claw hook construct at two segments above upper end vertebra on both sides followed by pedicle hook at upper end vertebra on the concave side and transverse process hook on convex side. Wisconsin wires were placed for the mid-thoracic segments. (c) 5 cases of CDM8 instrumentation were essentially same as the ISOLA constructs except the lumbar pedicle screws used were top loading monoaxial screws instead of vertebral screws connecting to rod with slotted connectors as in the ISOLA system. After placement of the fixation devices, curve reduction was made by manual pressure at apex and counter pressures at opposite ends under SSEP monitoring. Rod estimation and preliminary contouring was then made. Decortication of facet joints and transverse processes was made and bone grafts were placed at each decorticated facet joint. Instrumentation was then performed and final rod contouring made with insitu benders. Cell saver was used to retrieve blood and transfused back to patient intra-operatively. There were no neurological or wound complications. All AIS subjects were neurologically normal on detail clinical examination. Exclusion criteria included history of back injury, weakness or numbness in one or more limbs, urinary incontinence or nocturnal enuresis. None of the subjects had any history of pulmonary diseases and they were free from any respiratory symptoms or acute respiratory infection at the time of the MR studies before and after operation.

Ethical approval and informed consent for dynamic breath-hold MR imaging have been obtained from all the subjects and their parents.

\section{MRI assessment}

Pre operative MRI examination of the chest was performed in all subjects within two weeks before the operation, while the post operative MRI was performed after 6 months from the date of surgery. MRI examination was performed using a 1.5T MR scanner (Sonata, Siemens, Erlangen, Germany). The protocol of BH-MR imaging of the chest has been reported in previous published study [5]. In brief, fast gradient-recalled echo pulse sequence was used to obtain coronal images of the whole chest during full inspiration and expiration with parameters. The 
images were acquired in the supine position. All subjects were given clear instruction by experienced radiographers and practice took place before actual imaging. The subjects were instructed to fully inspire/fully expire and then hold their breaths at either full inspiration or full expiration. Scanning repeated three times for full inspiration and another three times for full expiration. Each breathhold scan took about 15-20 seconds, which was well tolerated by all subjects. The images with the maximum inspiratory and expiratory effort out of the three attempts were chosen for analysis.

Post-processing of the MR images was performed using a workstation (EasyVision, Philips Medical Systems, Best, the Netherlands). Volumetric measurements of total inspiratory and expiratory lung volume were determined by a semi-automated computerized segmentation method [6] (Fig 1). The MR images were also reformatted into axial and coronal planes so that motions of the chest wall and diaphragm could be assessed. The chest wall and diaphragmatic motions were measured in antero-posterior, left-right and cranio-caudal directions respectively. The chest wall diameters were measured at the level of the carina (Figure 2a) and at apex of the vertebral curve (Figure $2 \mathrm{~b})$ respectively. The chest wall dimensions were then measured as the largest anteroposterior (AP) and transverse (TS) dimensions on either side of the scoliosis sepa-

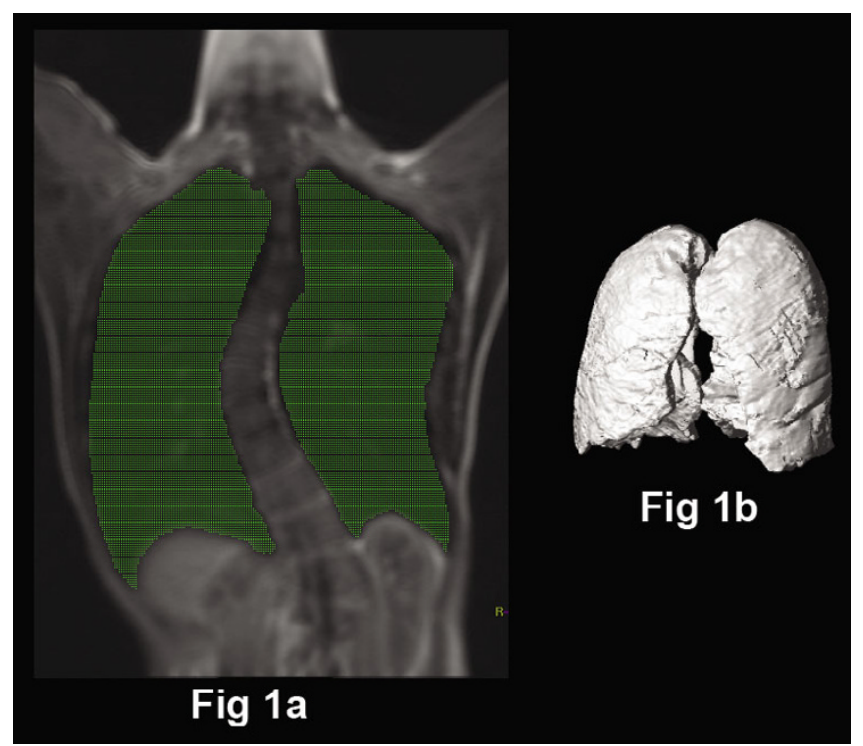

\section{Figure I}

Measurement of lung volumes by a semi-automated computerized method of delineating the lungs and summing crosssectional areas. (a) On the coronal image of the lung, threshold signal intensity is selected to highlight the air in green (lung). (b) The total lung volume is calculated by summating the volume of all coronal sections of the lungs from the front to the back of the body. rately. The diaphragmatic heights were taken as the vertical distance between the line drawn tangential to the highest point of the diaphragm and a line parallel to the lung apex (Fig 3a and 3b). All the lung volume, chest wall and diaphragmatic dimensions in the right and left hemithorax were measured separately, during both inspiration and expiration, and the differences were recorded.

Three measurements were made for each parameter by the same observer and the average value was taken for the analysis. Our previous studies showed high intraobserver and interobserver reliability on the MR measurements [5].

\section{Breathing Effort Assessment}

Patients were also asked to score their breathing effort before and after operation using a scale of 1-9 with ascending order of effort. Score 0 was equivalent to nonawareness of breathing effort at rest. Score 1-3 was equivalent of awareness of gentle breathing effort at rest with ascending order of effort. Score 4-6 was equivalent to feeling of breathlessness during exercise activities in ascending order of severity. Score 7-9 was equivalent to feeling of breathlessness at gentle exertion such as walking in ascending order of severity.

\section{Statistics}

The parameters in each group of subjects were expressed as median with inter-quartile range. Non-parametric Wilcoxon signed ranks test was used to compare the MR measurements before and after operation in all subjects.

Two-tailed probability values $<0.05$ were considered significant. SPSS for Windows statistical software (Release 13, SPSS Inc., Chicago, Illinois) was used in the analysis.

\section{Results}

The spatial resolution of the reformatted axial and coronal images of the chest was considered to be of diagnostic quality in all subjects. The fast image acquisition time allows good delineation of the lung volume, the chest wall and the diaphragm.

Surgical procedures in all patients were uneventful without significant complications. All patients were discharged home fully mobilized on average 11 days (range 9-15) from admission. Six month after operation, the median correction rate of Cobb's angle was 60\% (range $42 \%$ to $72 \%$ ). The median Cobb's angle changed from $59^{\circ}$ to $24^{\circ}$ while the sagittal Cobb's angle changed from $19.1^{\circ}$ to $23.1^{\circ}$.

The lung volumes, chest wall and diaphragmatic parameters before and after operation in all subjects are summarized in Table 2. 


\section{(a) at carina}

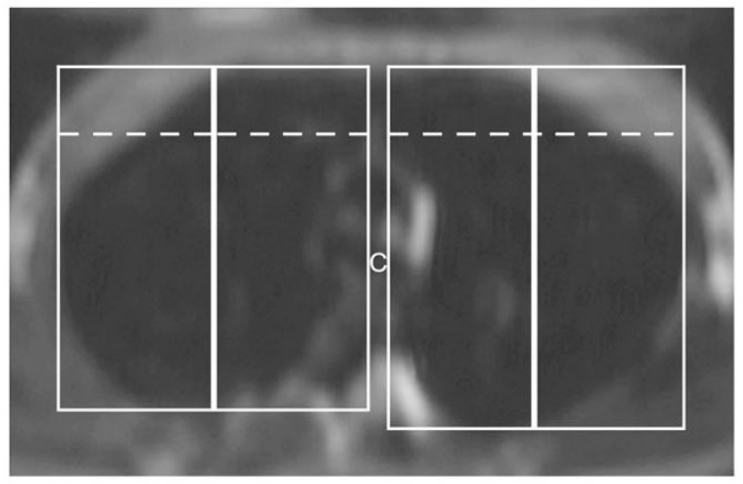

\section{(b) at vertebral apex}

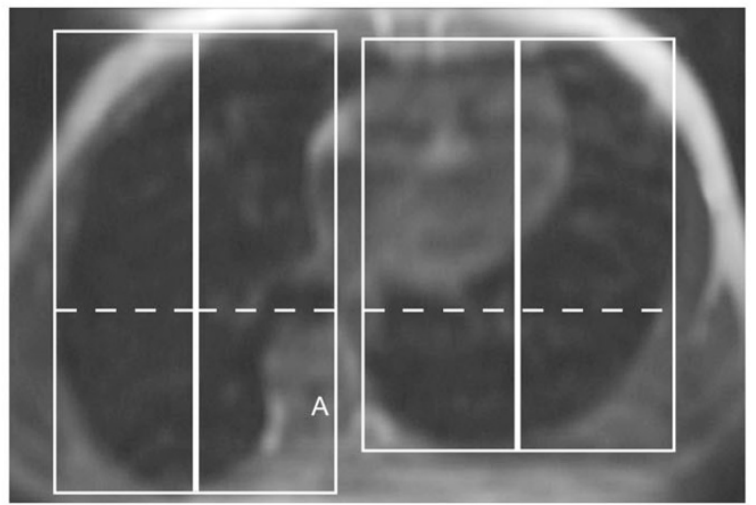

\section{Figure 2}

Measurement of AP and TS diameter of the chest wall on the reformatted axial image. (a) Upper level at the carina (C), maximal inspiratory image. (b) Lower level at the apical vertebra (A), maximal inspiratory image. Tangential lines are drawn to the anterior, posterior and lateral lung surfaces. The chest wall dimensions are then measured as the largest anteroposterior (AP, thick solid lines) and transverse (TS, dotted lines) dimensions on either side of the scoliosis separately. The chest wall motion is calculated as the difference between inspiration and expiration.

a

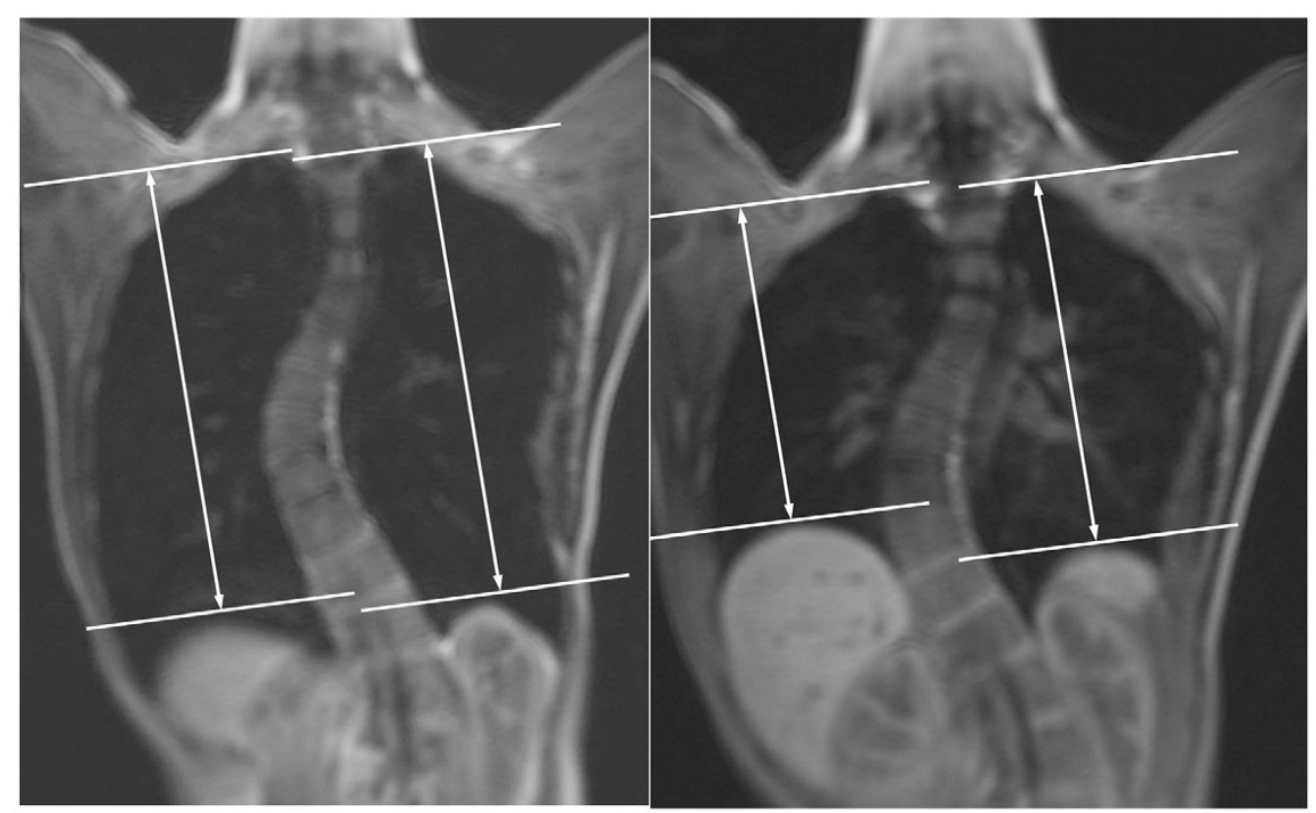

\section{Figure 3}

Measurement of diaphragmatic heights on the reformatted coronal image. (a) At maximal inspiratory image and (b) maximal expiratory image The diaphragmatic heights were taken as the vertical distance in between the line drawn tangent to the highest point of the diaphragm and a parallel line to the lung apex. The diaphragmatic motion is calculated as the difference between inspiration and expiration.

For lung volumes, the right lung (on the convexity side of 
Table 2: Lung, chest wall and diaphragmatic parameters, spinal curvatures in 16 subjects before and six months after corrective posterior spinal fusion. Figures are expressed as median (interquartile range)

\begin{tabular}{|c|c|c|c|}
\hline & Before surgery & After surgery & $P$ value (* significant at 0.05 level) \\
\hline \multicolumn{4}{|c|}{ Inspiratory volume (cc) } \\
\hline Right lung & $1719(1232,1858)$ & $1764(1362,1938)$ & 0.57 \\
\hline Left lung & $1392(1113,1607)$ & $1381(1152,1642)$ & 0.35 \\
\hline Total & $3127(2364,3464)$ & 3152 (2491, 3529) & 0.50 \\
\hline \multicolumn{4}{|c|}{ Expiratory volume (cc) } \\
\hline Right lung & $662(579,817)$ & $716(586,814)$ & 0.61 \\
\hline Left lung & $598(501,700)$ & $601(464,707)$ & 0.96 \\
\hline Total & $1269(1083,1498)$ & $1361(1038,1477)$ & 0.64 \\
\hline \multicolumn{4}{|c|}{ Change in lung volume (cc) } \\
\hline Right lung & $996(645,1126)$ & $969(698,1167)$ & 0.68 \\
\hline Left lung & $762(535,962)$ & $736(630,1018)$ & 0.22 \\
\hline Total & $1770(1206,2089)$ & $1730(1362,2203)$ & 0.30 \\
\hline \multicolumn{4}{|c|}{ Right lung AP diameter at carina (mm) } \\
\hline Inspiratory & $117(100,123)$ & $118(107,130)$ & 0.07 \\
\hline Expiratory & $84(75,92)$ & $88(82,96)$ & 0.06 \\
\hline Change & $29(21,42)$ & $29(21,42)$ & 0.98 \\
\hline \multicolumn{4}{|c|}{ Left lung AP diameter at carina $(\mathrm{mm})$} \\
\hline Inspiratory & $122(106,133)$ & $119(108,129)$ & 0.18 \\
\hline Expiratory & $89(80,99)$ & $93(87,101)$ & 0.41 \\
\hline Change & $29(16,46)$ & $27(18,33)$ & 0.14 \\
\hline \multicolumn{4}{|c|}{ Right lung AP diameter at apex (mm) } \\
\hline Inspiratory & $135(123,151)$ & $137(\mid 16,155)$ & 0.50 \\
\hline Expiratory & $1114(99,126)$ & $114(100,133)$ & 0.88 \\
\hline Change & $2 \mid(|5,3|)$ & $20(13,25)$ & 0.20 \\
\hline \multicolumn{4}{|c|}{ Left lung AP diameter at apex (mm) } \\
\hline Inspiratory & $126(118,145)$ & $126(121,138)$ & 0.92 \\
\hline Expiratory & $104(94,114)$ & $104(97,111)$ & 0.72 \\
\hline Change & $28(17,37)$ & $23(12,33)$ & 0.30 \\
\hline \multicolumn{4}{|c|}{ Right lung TS diameter at carina $(\mathrm{mm})$} \\
\hline Inspiratory & $97(80,116)$ & $111(106,116)$ & $0.013^{*}$ \\
\hline Expiratory & $85(75,93)$ & $87(75,96)$ & 0.30 \\
\hline Change & $15(3,27)$ & $28(17,33)$ & $0.006 *$ \\
\hline \multicolumn{4}{|c|}{ Left lung TS diameter at carina (mm) } \\
\hline Inspiratory & $92(79,103)$ & $104(99,110)$ & $0.002 *$ \\
\hline Expiratory & $79(59,84)$ & $86(76,93)$ & $0.044^{*}$ \\
\hline Change & $14(11,23)$ & $20(13,28)$ & 0.056 \\
\hline \multicolumn{4}{|c|}{ Right lung TS diameter at apex (mm) } \\
\hline Inspiratory & $106(85,126)$ & $114(105,120)$ & $0.039 *$ \\
\hline Expiratory & $87(71,92)$ & $91(84,100)$ & $0.034^{*}$ \\
\hline Change & $16(10,31)$ & $24(13,32)$ & 0.796 \\
\hline \multicolumn{4}{|c|}{ Left lung TS diameter at apex (mm) } \\
\hline Inspiratory & $112(93,117)$ & $113(104,122)$ & 0.088 \\
\hline Expiratory & $98(68,113)$ & $100(83,112)$ & 0.163 \\
\hline Change & $9(6,13)$ & $12(7,17)$ & 0.301 \\
\hline \multicolumn{4}{|c|}{ Right Diaphragmatic height (mm) } \\
\hline Inspiratory & $185(172,202)$ & $201(182,223)$ & $<0.01 *$ \\
\hline
\end{tabular}


Table 2: Lung, chest wall and diaphragmatic parameters, spinal curvatures in 16 subjects before and six months after corrective posterior spinal fusion. Figures are expressed as median (interquartile range) (Continued)

\begin{tabular}{|c|c|c|c|}
\hline Expiratory & $136(129,150)$ & $146(135,156)$ & 0.196 \\
\hline Change & $49(25,65)$ & $53(44,66)$ & $0.039 *$ \\
\hline \multicolumn{4}{|c|}{ Left Diaphragmatic height (mm) } \\
\hline Inspiratory & $185(172,206)$ & $198(186,215)$ & $0.01 *$ \\
\hline Expiratory & $142(129,159)$ & $148(136,161)$ & 0.234 \\
\hline Change & $44(33,57)$ & $53(44,66)$ & $0.039 *$ \\
\hline Breathing effort & $4(1,4)$ & $1.75(I, 3)$ & $0.012 *$ \\
\hline Coronal Cobb angle (MR) & $59(53,64)$ & $24(21,28)$ & $<0.01 *$ \\
\hline Axial angle (MR) & $9.9(4.7,13.0)$ & $9.6(4.0,14.7)$ & 0.215 \\
\hline Sagittal angle (MR) & $19.1(13.1,28.8)$ & $23.1(\mid 7.2,31.2)$ & 0.056 \\
\hline
\end{tabular}

the scoliotic curve) and total inspiratory and expiratory lung volume showed slight but insignificant increase after operation. Neither right/left lung nor the total vital lung capacity (defined as the difference in lung volume between inspiration and expiration) differ significantly when comparing the pre-operative with the post-operative study.

For the TS diameter of bilateral chest wall, there was significant increase in baseline value on both right and left lung at either carina level or apical vertebral level during both inspiration and expiration. When the difference between inspiration and expiration was considered, the lateral chest wall movement at carina level was improved significantly on right/convex side $(\mathrm{p}=0.013)$ while marginally significant on left/concave side $(\mathrm{p}=0.056)$. but there was no statistically significant change at the apical vertebra level.

For the AP diameter of bilateral chest wall, there was no significant difference on either right lung or left lung in between operation. The AP chest wall movement also showed no significant interval change.

For the diaphragmatic heights, they were significantly increased on both right and left sides during inspiration while the absolute value during expiration was unchanged and hence the motion of the diaphragms, defined as the difference in diaphragmatic height between inspiration and expiration, was significantly increased after operation when compared with the pre-operative stage.

The median breathing effort reported by the subjects before operation was 4 (range 1-9) while the median breathing effort after operation was 1.75 (range 1-5).

In summary, there was improvement of lateral chest wall and diaphragmatic motions in AIS subjects six months after posterior spinal fusion. Lung volumes however, did not show significant increase after operation at this stage.

\section{Discussion}

Though pulmonary function impairment has long been reported in AIS patients, it is difficult to measure and describe respiratory motions because of the limited methods available for real time dynamic assessment. In the present and previous published study, we have demonstrated that by using multi-planar reformat technique, coronal and axial sectional planes could be obtained simultaneously with measurement of lung volumes during a single inspiration and expiration movement. This MR technique has also been validated which showed significant positive correlations with plethysmography parameters[6]. We therefore propose the use of dynamic breath-hold MR as a novel non-invasive tool for clinical analysis of lung volume, diaphragmatic and chest wall motion in AIS patients.

In this study, dynamic BH-MR imaging has been used to compare the pre and post operative lung function changes in AIS patients after spinal fusion. We found that the metallic implants only caused mild distortion artefacts on the immediate adjacent structures such as the vertebral column and the central canal; while the visualization of the lungs, the chest walls and diaphragms were not affected.

In the literature, there has been debate about the effect of surgical correction on lung function. Many authors have written that scoliosis correction definitely improves measured pulmonary functions [7], such as vital capacity. Others disagree and declare that pulmonary functions remain essentially unchanged [8-10] or even becoming worsen[11].

In the previous published study, we found that the chest wall and diaphragmatic motion in AIS patients were not restricted, i.e. the chest wall and the diaphragmatic motions were as mobile as those in the normal subjects and therefore there was no suggestion of neuromuscular dysfunction in AIS [5]. 
In the current study, we found that the chest wall motion and diaphragmatic motions could be further improved after posterior spinal surgery. This was consistent with the subjective feeling of reducing breathing effort after operation as reported in this group of patients. The reason for increased amplitude of lateral chest wall movement could be explained by the less distorted chest wall configuration after surgery, while the improvement of diaphragmatic excursion could be the combined effect of chest wall remodeling, lessen compressive effect by both the scoliotic spine and the rotated mediastinum as well as improvement in degree of hypokyphosis after surgery.

The absolute lung volumes however, did not show significant increase in this cohort. As the current study was carried out shortly after the operation within six months, longer follow up study is warranted which might show a change in pulmonary volume.

Dynamic MR is considered as a promising investigation tool in assessing respiratory mechanism in the AIS group. It might be useful for both short and long term follow up of pulmonary function of AIS patients, in particular, for assessing post operative changes.

\section{Conclusion}

With the application of ultrafast dynamic BH-MR imaging and multi-planar reformat technique, the lung volume, chest wall and diaphragmatic motion between inspiration and expiration could now be accurately measured with high reproducibility in AIS patients. Improvement of lateral chest wall and diaphragmatic motions are evident in AIS patients six months after posterior spinal fusion. BHMR might be sued for long term post operative assessment of pulmonary function in AIS patients.

\section{Abbreviations}

AIS: Adolescent Idiopathic Scoliosis

\section{BH: breath-hold}

\section{MR: Magnetic Resonance}

\section{Competing interests}

The author(s) declare that they have no competing interests.

\section{Authors' contributions}

All the authors have contributed to conception and design of the manuscript. Particularly, WCWC and BKWN carried out the analysis of data and wrote the manuscript; AML and TPL contributed to the editing of the manuscript, clinical recruitment and assessment; WWML participated in the editing of the manuscript; JCYC contributed to the editing of the manuscript and secured funding. All authors have read and approved the final manuscript.

\section{References}

I. Leaver JM, Alvik A, Warren MD: Prescriptive screening for adolescent idiopathic scoliosis: a review of the evidence. Int J Epidemiol 1982, I I(2): I0 I-III.

2. Gagnon S, Jodoin A, Martin R: Pulmonary function test study and after spinal fusion in young idiopathic scoliosis. Spine 1989 , I 4(5):486-490.

3. Muirhead A, Conner AN: The assessment of lung function in children with scoliosis. J Bone Joint Surg Br 1985, 67(5):699-702.

4. Kotani T, Minami S, Takahashi K, Isobe K, Nakata Y, Takaso M, Inoue M, Maruta T, Akazawa T, Ueda T, Moriya $H$ : An analysis of chest wall and diaphragm motions in patients with idiopathic scoliosis using dynamic breathing MRI. Spine 2004, 29(3):298-302.

5. Chu WC, Li AM, Ng BK, Chan DF, Lam TP, Lam WW, Cheng JC: Dynamic magnetic resonance imaging in assessing lung volumes, chest wall, and diaphragm motions in adolescent idiopathic scoliosis versus normal controls. Spine 2006, 3 I ( I 9):2243-2249.

6. Gierada DS, Hakimian S, Slone RM, Yusen RD: MR analysis of lung volume and thoracic dimensions in patients with emphysema before and after lung volume reduction surgery. AJR Am J Roentgenol 1998, I 70(3):707-7|4.

7. Kinnear WJ, Johnston ID: Does Harrington instrumentation improve pulmonary function in adolescents with idiopathic scoliosis? A meta-analysis. Spine 1993, I 8( I I): I 556-1559.

8. Shneerson JM, Edgar MA: Cardiac and respiratory function before and after spinal fusion in adolescent idiopathic scoliosis. Thorax 1979, 34(5):658-66I.

9. Upadhyay SS, Ho EK, Gunawardene WM, Leong JC, Hsu LC: Changes in residual volume relative to vital capacity and total lung capacity after arthrodesis of the spine in patients who have adolescent idiopathic scoliosis. J Bone Joint Surg Am 1993, 75(I):46-52.

10. Zorab PA, Prime FJ, Harrison A: Lung function in young persons after spinal fusion for scoliosis. Spine 1979, 4(I):22-28.

II. Kinnear WJ, Kinnear GC, Watson L, Webb JK, Johnston ID: Pulmonary function after spinal surgery for idiopathic scoliosis. Spine 1992, I7(6):708-7|3.

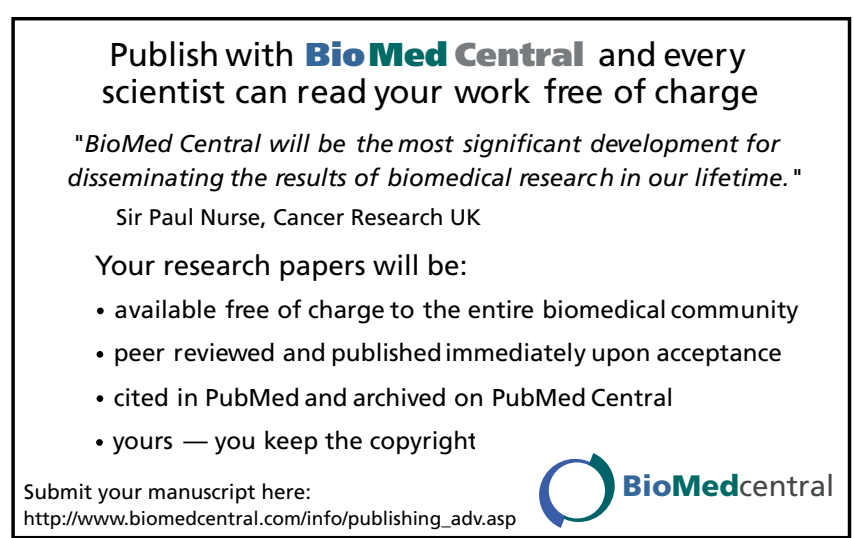

